Copyright (C) 2014 IEEE. Personal use of this material is permitted. Permission from IEEE must be obtained for all other uses, in any current or future media, including reprinting/republishing this material for advertising or promotional purposes, creating new collective works, for resale or redistribution to servers or lists, or reuse of any copyrighted component of this work in other works. 


\section{A Compact Robotic Device for Upper-Limb Reaching Rehabilitation}

\author{
Lei Cui, Ze Ru Eng, Jeremy Devene, and Tele Tan \\ School of Civil and Mechanical Engineering \\ Curtin University \\ Perth, Australia \\ lei.cui@curtin.edu.au
}

\author{
Garry Allison \\ School of Physiotherapy and Exercise Science \\ Curtin University \\ Perth, Australia \\ g.allison@curtin.edu.au
}

\begin{abstract}
This paper presents a compact linear-motion robotic device for upper-extremity reaching rehabilitation. Starting from conceptual design, the paper describes electronic circuit design and program development. The work develops a prototype that provides active and passive rehabilitation training. In active training, subjects actively move their arm with assistive or resistive force from the device to finish predefined displacement and force profiles. In passive training, subjects remain passive while the device moves the limb following the predefined displacement profile. Engineering specifications with adequate safety factor are determined and standard electronic and readily available mechanical components are exploited to keep the total cost low.
\end{abstract}

Keywords-Rehablitation, Robotics, Upper-Extrimity, Variable Stiffness Actuation

\section{INTRODUCTION}

Stroke is the leading cause of severe disabilities in the developed world. The prevalence of stroke in Australia is about $110 / 10,000$ and the societal and economical costs are estimated to be about $\$ 2.14$ billion per year [1]. Being a common outcome of stroke, hemiplegia affects muscles of the face, arm and leg on one side and prevents patients from doing a variety of activities, ranging from walking to feeding themselves. Substantial gain in hemiplegic patients' healthrelated quality of life can be achieved by ensuring continuous stroke therapy and rehabilitation [2].

Various clinical trials have demonstrated that intensity and task specificity are the main drivers in an effective treatment program after stroke [3, 4]. Highly repetitive movement training can result in improved recovery $[4,5]$. Patients with upper-arm disabilities most often require gradual but consistent and extensive physical therapy. The exercises are often simple but repetitive and require full assistance from a physical therapist, whose consistent presence not only has distinctively negative social and economic effects, but also increases the sense of dependence and sometimes even leads to depression in patients [6].

Robot-assisted movement training is fast becoming a common feature in rehabilitation [7, 8]. Robotic devices have the programmable force-producing ability, leading to realistic replication of the features of a therapist's assistance. Moreover, robotic devices can easily apply new constraints and optimize the required movement patterns, which might not be feasible with therapists. Therefore, complex motor-learning tasks can be generated far more precisely with robotic devices than in conventional treatment approaches.

Since the pioneering study of MIT-Manus [9], the number of research groups developing robotic therapy devices has rapidly increased, and various devices have been developed for after-stroke automating training for the upper extremity. Current robotic rehabilitation devices can be classified into two categories: end-effector interacting devices and exoskeletal devices.

In the first category, patients' arm is bound to the robot end-effector and moves with it. For instance, the ARM Guide enables hand to move along a linear rail to help shoulder and elbow to recover [10]. MIME system employs two 6R Puma industry robot arms and predefined 12 targeted reaching tasks [11]. GENTLE/s requires patients to reach and touch virtual objects presented in virtual environment for shoulder and elbow rehabilitation [12]. Bi-Manu-Track enables hemi-paretic patients to bilaterally practice two different movement cycles: forearm pronation/supination and wrist flexion/extension [13].

The exoskeletal devices in the second category have rigid links attached to the human arm and motors at exoskeletal joints. For example Armin [14], MGA [15], MAHI [16], Armeo Spring [17], WREX [18], BONES [19]. This approach introduces large mass to, and subsequently changes the dynamics of, a human arm.

The initial results of robot-assisted rehabilitation are promising: patients who receive more therapy with a robotic device recover more movement ability [20-22]

Comparing with healthy people, hemiplegic patients undergo abnormal synergistic muscle activations, affecting limb mechanics and changing limb impedance due to the modification of tixotropic tissue-characteristics [23, 24].

This work aims to design, develop and produce a compact upper-extremity hemiplegia rehabilitation robot that can measure impedance during movements by exploiting low-cost electronic and mechanical components. This robotic device is equipped with a light-weight mobile platform and sensitive load cells that can measure slight hand motion and provide active and passive assistance. 
This compact robotic device presents several advantages. First, the device is antagonistically actuated to provide inherent safety and variable stiffness and thus is able to accommodate the need of patients with various arm stiffness. Second, it is light and compact and can be positioned at different angles and positions to augment different reaching tasks either in a clinic or at home. Third, it is able to measure force-feedback with low-cost load cells via novel mechanism design, reducing the total cost considerably.

The paper is organized as follows. Section II introduces the overall design of the device. Section III presents the endeffector with force sensing capacity via low-cost load cells activated by a novel lever linkage. Section IV calculates the maximum loading on the end-effector and lays the ground for motor selection and part stress analysis. Section V discusses the antagonistic actuation and Section VI concludes the paper.

\section{MECHANICAL DESIGN}

The robotic device is to conduct active and passive rehabilitation sessions with patients. In active sessions, the device is involved in helping the patients finish the predefined displacement, velocity, and force profiles by applying a certain assistive or resistive force when the individuals with hemiplegia actively move the arm in keeping with their recovery condition. The main function of the active movement is to generate low-load movement and force/position training. In passive sessions, the individuals with hemiplegia remain passive while the device exercises the pre-defined displacement by moving the arm via the end-effector.

To realize the two modes, the patients are to hold on to the end-effector to conduct rehabilitation session. The device detects and records the patient's performance, which is to be reviewed by the therapist.

The device is designed such that a linear guide rail is used to provide linear motion training to the patient, as in Fig. 1. An end-effector integrated with an arm rest and force sensor is attached to the sliding block of the linear guide rail. Two motors are used to actuate the end-effector through stainlesssteel cables. The motors are fixed on a spring mounted base to provide a force and movement buffer during operation: this antagonistic actuation yields the capacity of adjusting the stiffness of the platform. This device is programmed such that therapists can choose between active and passive modes. If active mode is chosen, the resultant displacement, velocity, and force profiles applying on to the user are required to be input by the physiotherapist.

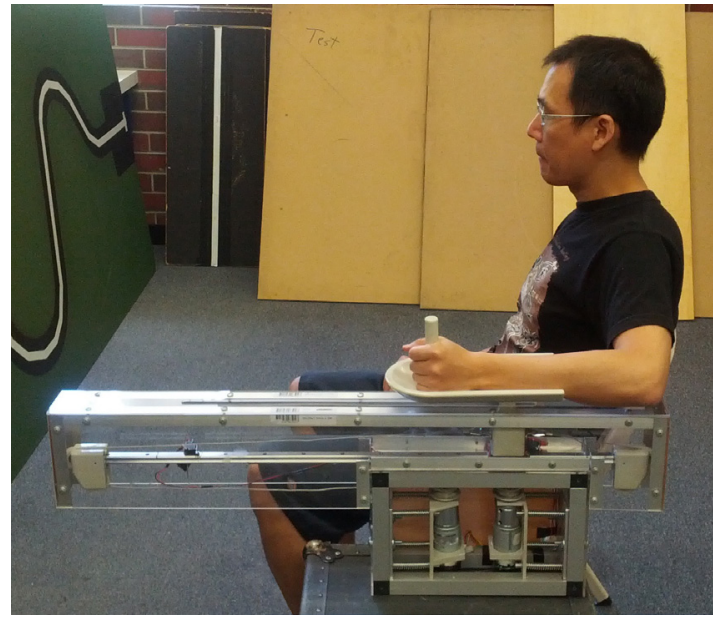

Figure 1 An overview of the robotic device

In terms of safety, passive safety features such as mechanical stops are used at both ends of the rail and an active emergency stop is implemented to cut the power supply.

A modular design is adopted for the device, which consists of end-effector, guiderail, chassis, cable-routing, and actuation modules, as in Fig. 2.

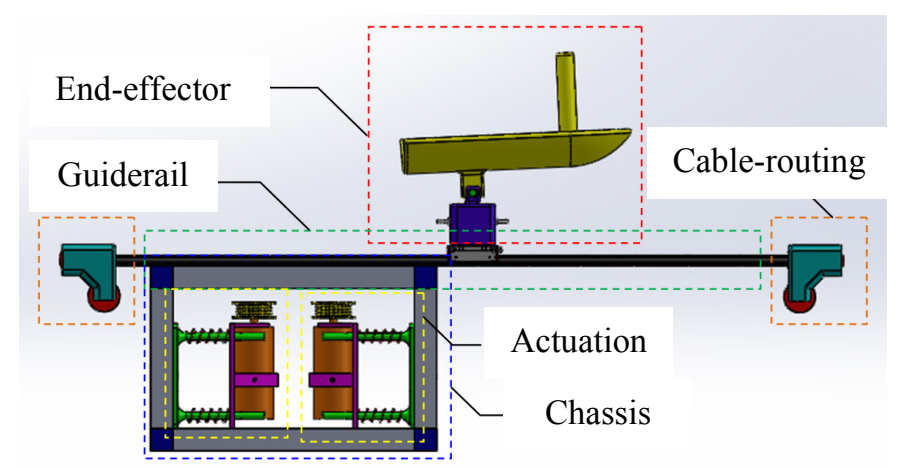

Figure 2 The modules of the robotic device

\section{END-EFFECTOR WITIH FORCE SENSING}

Force-sensing is an integrated part of the majority of robotic rehabilitation platforms. However, the high-price tag of many sensitive force/torque sensors prevents such devices from being employed economically. This work overcomes this impediment by using a lever mechanism to effectively enlarge the force at the end-effector.

Finding a load cell that comes with high performance and small in size at a low cost presents a challenge. The selected FX1901 compression load cell [25] is the smallest among the range that provides relatively high accuracy: $\pm 1 \%$ of its measuring range at a low cost (\$40 each), as shown in Fig. 3. 


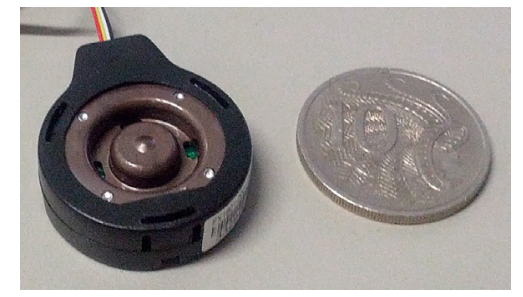

Figure 3 The FX1901 compression load-cell

The maximum force measurable by the force sensor is $11.34 \mathrm{~kg}$, thus the accuracy of the encoder is $0.1 \mathrm{~kg}$. An INA $125 \mathrm{P}$ instrumentation amplifier is paired with the load cell such that that the analog output signals is readable by the Arduino Due microcontroller, as in Fig. 4.

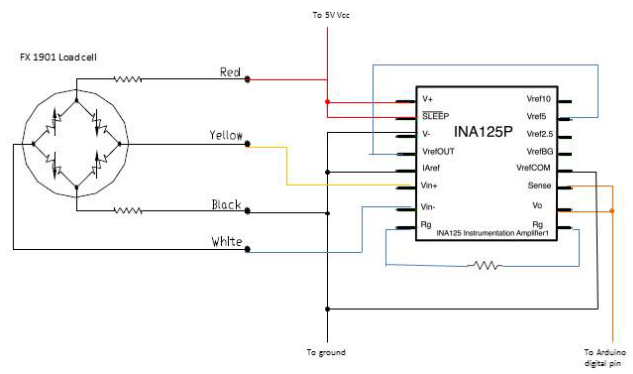

Figure 4 Schematic diagram of load cell with amplifier

A thorough calibration of the load cell is conducted: the readings from both load cells are linear and the minimum weight to detectable by force sensor is approximately $110 \mathrm{~g}$.

The rather high minimum sensitivity poses a challenge to mechanical design. This is solved by using a lever mechanism, which is embedded into the end-effector design, as in Fig. 5.

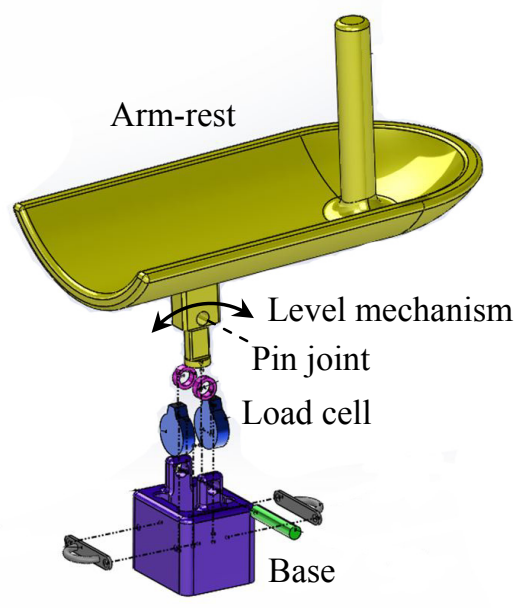

Figure 5 The end-effector effectively enlarging the force

The end-effector consists of an arm-rest, a load-cell compartment, and a base. The arm rest provides ample space to comfortably support a human forearm and a handle helps to stabilize the hand. With a pin-joint below the rest, it also serves as a lever mechanism to effectively amplify the force at the arm-rest. The load-cell compartment accommodates two load-cells to measure the end-effector force: the bottom cylinder is aligned to the centers of the load-cells. The base is fixed on to the linear guiderail-slide block with two stainless steel saddle attached to its front and back to move the arm with minimum backlash.

\section{LOADING ON THE PLATFORM}

This section considers the longitudinal direction loading, i.e., along the guiderail direction, of the end-effector. The applied vertical force is assumed by considering the following factors: average weight of the human upper limb, the estimated weight of the instruments on the linear guide rail slide block, the friction between the carrier block and the rail, the maximum acceleration of a human arm, and the maximum pull/push force of a human arm.

Longitudinal load determined the force required to move the end-effector forward and backward. Since there is no information on axial strength of the upper-extremity hemiplegic patients, strength of a healthy subject is used for calculation according to the research done by The Ergonomic Center of North Carolina [26].

The load applied on to the cable is an important piece of information. It not only determines the required strength and type of the cable, but also the strength of the pulleys and the torque of the motors.

To determine the maximum tension on the cable, the cable is designed to handle weigh such that it will accelerate the arm at the intended maximum acceleration $\left(2.5 \mathrm{~m} / \mathrm{s}^{2}\right)$. The force analysis in the direction of motion is shown in Fig. 6.

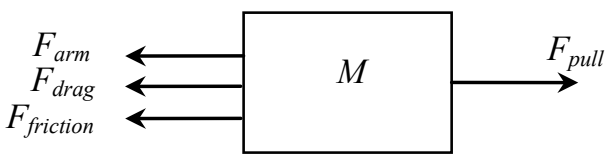

Figure 6 Free body diagram of carriage block while moving along the rail

The forces can be calculated using the following equation:

$$
M \ddot{x}=\sum F_{e x t}=F_{p u l l}-F_{\text {arm }}-F_{\text {drag }}-F_{\text {friction }}
$$

According to the preliminary normative study performed in by [27], the average mass of the arm weights at $4 \mathrm{~kg}$. The mass of instruments fixed on the slide block is $0.8 \mathrm{~kg}$. This gives the total mass $M 4.8 \mathrm{~kg}$. The maximum arm resistant force is estimated to be $44 \mathrm{~N}$, the drag force is set to be $5 \%$ of the pull force, and the friction coefficient between aluminum is 1.05. This yields the maximum pull force at $64.25 \mathrm{~N}$. 


\section{TENDON-BAsed ANTAGNistic ACUTATION}

One of the unique aspects of rehabilitation robots is that the patient fills the role of both patient and operator. This makes rehabilitation robots safety critical systems, and no single failure can cause a hazard. A safe rehabilitation robot does not allow uncontrolled motions and excessive force on patients. On the other hand, as force-applying devices, rehabilitation robots are required to accurately reproduce the position/force profile.

This work adopts the intrinsically safe variable stiffness actuators (VSAs) [28] to address the safety issue. When coupled with compensation by control, the VSA is able to accurately control the position and force.

Variable impedance control of VSAs is the hallmark of the biological motor control, which adapts the impedance of the overall biomechanical system to different task requirements and stochastic disturbance. A transfer of this principle to robotics is to connect two actuators antagonistically to control the position and impedance simultaneously, as in Fig. 7.

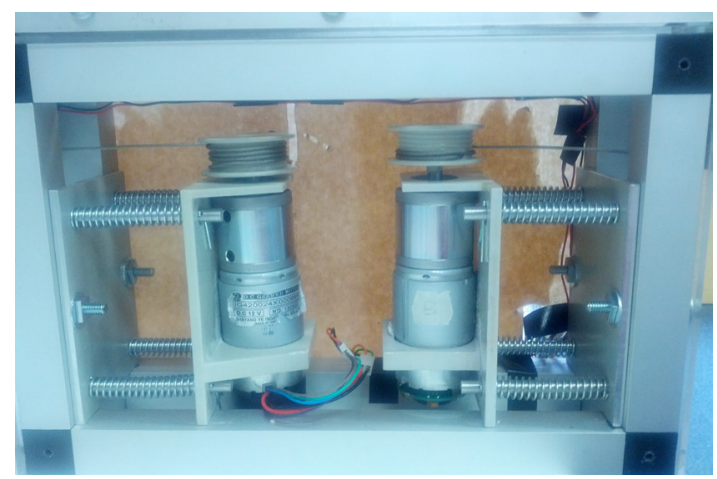

Figure 7 The antagonistic actuation

The motor mounting holds the electric motor in place and eight pre-tensioned springs between the motor mountings and the base creates a buffer to absorb shock forces such as sudden step increase of torque from the motor as well as adding on flexibility to the system so that the patients are able to operate the device more comfortably.

Commercial off-the-shelf components with adequate performance are employed in the design. Two geared carbonbrush DC motor with attached rotary encoder as actuator, two 10A Motor Driver Shields, and an Arduino Due microcontroller.

An Arduino program is developed such that it ensures all the sensors are operative and calibrates the system prior to further use of the device. Besides that, it also contains the codes that carry out assistive and passive rehabilitation. PID controllers are used to for positioning control and force feedback control.

The control is separated into master and slave loops, where the master loop regulates the motor that pulls the end effector and the slave loop regulates the other motor to maintain the position and cable tension, realizing position/force control. Force data and encoder position are sent to the $\mathrm{PC}$ via serial port, allowing real-time monitoring and recording of the patient's performance.

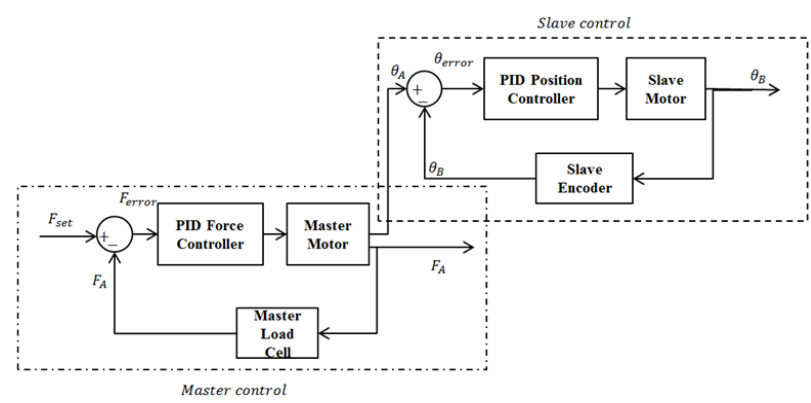

Figure 8 Control loop

\section{FUTURE WORK}

The first stage of future work will improve the overall compactness of the device and reduce the weight of the endeffector. This entails moving the load cells to the cable routing sheave pulleys and routing the cable over a spring loaded sheave block, in order to improve the devices' response, as in Fig. 9.

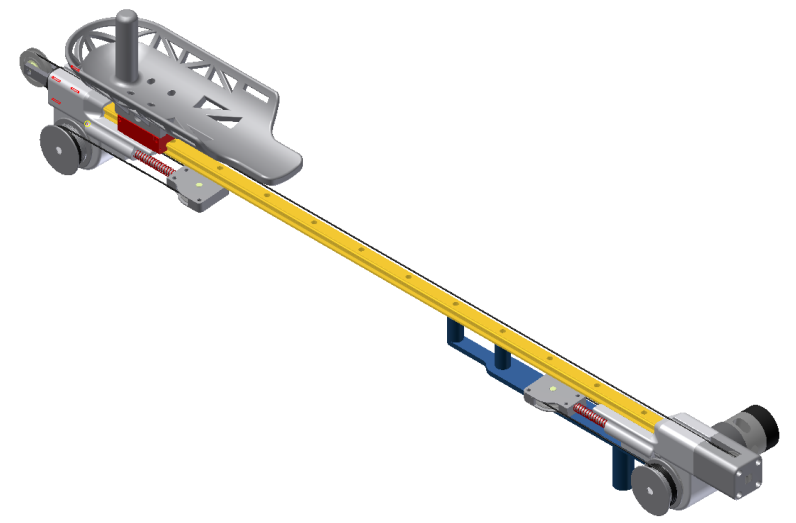

Figure 9 The improved Combot

The second stage will focus on getting the end effecter to move in a three dimensional space. This involves mounting the current device onto a rotary platform to develop a costeffective system. The forward and inverse kinematics [29], dynamics, and control will be investigated.

\section{CONCLUSIONS}

This work presented a compact robotic device for upperlimb reaching rehabilitation. The device consisted of several modules: end-effector, guiderail, cable-routing, actuation, and chassis. The end-effector served as an arm-rest and contained a load-cell compartment and a novel lever mechanism that effectively amplified the force at the arm-rest. The antagonistic actuation provided intrinsic safety and could vary the stiffness to accommodate patients' different requirements.

\section{ACKNOWLEDGMENT}


The first author would like to thank the Startup Fund from Curtin University and the insightful suggestions from Professor Thickbroom at the Australian Neuro-Muscular Research Institute (ANRI).

\section{REFERENCES}

[1] D. A. Cadilhac, R. Carter, A. G. Thrift, and H. M. Dewey, "Estimating the Long-Term Costs Of Ischemic and Hemorrhagic Stroke for Australia," Stroke, vol. 40, pp. 915-921, March 1, 20092009.

[2] W. M. Hopman and J. Verner, "Quality of Life During and After Inpatient Stroke Rehabilitation," Stroke, vol. 34, pp. 801-805, March 1, 20032003.

[3] R. P. Van Peppen, G. Kwakkel, S. Wood-Dauphinee, H. J. Hendriks, P. J. Van der Wees, and J. Dekker, "The impact of physical therapy on functional outcomes after stroke: what's the evidence?," Clinical Rehabilitation, vol. 18, pp. 833-862, August 1, 2004 2004.

[4] K. Gert, K. Boudewijn, and L. Eline, "Understanding the pattern of functional recovery after stroke: Facts and theories," Restorative Neurology and Neuroscience, vol. 22, pp. 281-299, 2004.

[5] C. Bütefisch, H. Hummelsheim, P. Denzler, and K.H. Mauritz, "Repetitive training of isolated movements improves the outcome of motor rehabilitation of the centrally paretic hand," Journal of the Neurological Sciences, vol. 130, pp. 59-68, 1995.

[6] M. Eriksson, K. Asplund, E.-L. Glader, B. Norrving, B. Stegmayr, A. Terént, et al., "Self-Reported Depression and Use of Antidepressants After Stroke: A National Survey," Stroke, vol. 35, pp. 936-941, April 1, 20042004.

[7] G. Kwakkel, B. J. Kollen, and H. I. Krebs, "Effects of Robot-Assisted Therapy on Upper Limb Recovery After Stroke: A Systematic Review," Neurorehabilitation and Neural Repair, vol. 22, pp. 111-121, March/April 20082008.

[8] K. Zhang, J. S. Dai, and Y. Fang, "Constraint Analysis and Bifurcated Motion of the 3PUP Parallel Mechanism," Mechanism and Machine Theory, vol. 49, pp. 256-269, 2012.

[9] N. Hogan, H. I. Krebs, J. Charnnarong, P. Srikrishna, and A. Sharon, "MIT-MANUS: a workstation for manual therapy and training. I," in Robot and Human Communication, 1992. Proceedings., IEEE International Workshop on, 1992, pp. 161-165.

[10] D. J. Reinkensmeyer, C. D. Takahashi, W. K. Timoszyk, A. N. Reinkensmeyer, and L. E. Kahn, "Design of robot assistance for arm movement therapy following stroke," Advanced Robotics, vol. 14, pp. 625-637, 2001/01/01 2001.

[11] P. S. Lum, C. G. Burgar, M. Van der Loos, P. C. Shor, M. Majmundar, and R. Yap, "The MIME robotic system for upper-limb neuro-rehabilitation: results from a clinical trial in subacute stroke," in Rehabilitation Robotics, 2005. ICORR 2005. 9th International Conference on, 2005, pp. 511-514.

[12] R. Loureiro, F. Amirabdollahian, M. Topping, B. Driessen, and W. Harwin, "Upper Limb Robot Mediated Stroke Therapy-GENTLE/s Approach," Autonomous Robots, vol. 15, pp. 35-51, 2003.

[13] S. Hesse, H. Schmidt, and C. Werner, "Machines to support motor rehabilitation after stroke: 10 years of experience in Berlin," Journal of Rehabilation Research \& Develepment, vol. 43, pp. 671-678, 2006.

[14] T. Nef and R. Riener, "ARMin - design of a novel arm rehabilitation robot," in Rehabilitation Robotics, 2005. ICORR 2005. 9th International Conference on, 2005, pp. 57-60.

[15] C. Carignan, M. Liszka, and S. Roderick, "Design of an arm exoskeleton with scapula motion for shoulder rehabilitation," in Advanced Robotics, 2005. ICAR '05. Proceedings., 12th International Conference on, 2005, pp. 524-531.

[16] A. Gupta and M. K. O'Malley, "Design of a haptic arm exoskeleton for training and rehabilitation," Mechatronics, IEEE/ASME Transactions on, vol. 11, pp. 280-289, 2006.

[17] J. Zariffa, N. Kapadia, J. L. K. Kramer, P. Taylor, M. Alizadeh-Meghrazi, V. Zivanovic, et al., "Effect of a robotic rehabilitation device on upper limb function in a sub-acute cervical spinal cord injury population," in Rehabilitation Robotics (ICORR), 2011 IEEE International Conference on, 2011, pp. 1-5.

[18] A. Frisoli, F. Salsedo, M. Bergamasco, B. Rossi, and M. C. Carboncini, "A force-feedback exoskeleton for upper-limb rehabilitation in virtual reality," Applied Bionics and Biomechanics, vol. 6, pp. 115-126, 2009/07/27 2009.

[19] J. Klein, S. J. Spencer, J. Allington, K. Minakata, E. T. Wolbrecht, R. Smith, et al., "Biomimetic orthosis for the neurorehabilitation of the elbow and shoulder (BONES)," in Biomedical Robotics and Biomechatronics, 2008. BioRob 2008. 2nd IEEE RAS \& EMBS International Conference on, 2008, pp. 535541 .

[20] H. Neville and I. K. Hermano, "Interactive robots for neuro-rehabilitation," Restorative Neurology and Neuroscience, vol. 22, pp. 349-358, 2004.

[21] S. Hesse, G. Schulte-Tigges, M. Konrad, A. Bardeleben, and C. Werner, "Robot-assisted arm trainer for the passive and active practice of bilateral forearm and wrist movements in hemiparetic subjects," Archives of physical medicine and rehabilitation, vol. 84, pp. 915-920, 2003.

[22] A. C. Lo, P. D. Guarino, L. G. Richards, J. K. Haselkorn, G. F. Wittenberg, D. G. Federman, et al., "Robot-Assisted Therapy for Long-Term UpperLimb Impairment after Stroke," New England Journal of Medicine, vol. 362, pp. 1772-1783, 2010. 
[23] D. Piovesan, M. Casadio, F. A. Mussa-Ivaldi, and P. G. Morasso, "Multijoint arm stiffness during movements following stroke: Implications for robot therapy," in Rehabilitation Robotics (ICORR), 2011 IEEE International Conference on, 2011, pp. 1-7.

[24] J. J. Palazzolo, M. Ferraro, H. I. Krebs, D. Lynch, B. T. Volpe, and N. Hogan, "Stochastic Estimation of Arm Mechanical Impedance During Robotic Stroke Rehabilitation," Neural Systems and Rehabilitation Engineering, IEEE Transactions on, vol. 15, pp. 94103, 2007.

[25] FX1901 Series. (2013). Available: http://www.measspec.com/product/t product.aspx?id=2446

[26] The Ergonomic Center of North Carolina. (2006, April 15, 2013). Human Strength Data Tables 13.

[27] L. Colizzi, A. Lidonnici, and L. Pignolo, "The Aramis Project: A Concept Robot and Technical
Design," Journal of Rehabilitation Medicine, vol. 41, pp. 1011-1015, // 2009.

[28] D. Gan, N. G. Tsagarakis, J. S. Dai, D. G. Caldwell, and L. Seneviratne, "Stiffness Design for a Spatial Three Degrees of Freedom Serial Compliant Manipulator Based on Impact Configuration Decomposition," Journal of Mechanisms and Robotics, vol. 5, pp. 011002-011002, 2012.

[29] L. Cui and J. S. Dai, "Reciprocity-Based Singular Value Decomposition for Inverse Kinematic Analysis of the Metamorphic Multifingered Hand," $A S M E$ Journal of Mechanisms and Robotics, vol. 4, pp. 034502-6, 2012. 